\title{
AVALIAÇÃO DE PLATAFORMA DE SISTEMA DE ARQUIVOS DISTRIBUÍDOS EM CLUSTER
}

\author{
Gledson de Oliveira' $^{1}$; Angelo Amancio Duarte ${ }^{2}$; \\ 1. Bolsista PIBIC/CNPq, Graduando em Engenharia da Computação, Universidade Estadual de Feira de Santana, \\ e-mail: gleddson.1@gmail.com \\ 2. Orientador, Departamento de Tecnologia, Universidade Estadual de Feira de Santana, e-mail: \\ angeloduarte@uefs.br
}

PALAVRAS-CHAVE: Sistema de Arquivos Distribuídos; Análise de Dados; Alto desempenho.

\section{INTRODUÇÃO}

Análise de sentimentos (Ou também, opinion mining,) é um campo da ciência que estuda a opinião, o comportamento, o sentimento dentre outros aspectos que as pessoas utilizam na comunicação, por exemplo (BARBOSA, 2012). Estudo de sentimentos sobre as informações publicadas no twitter tem sido amplamente explorados e aplicados na detecção de características, como determinar se um comentario foi positivo ou negativo e podem ser utilizadas para fins estratégicos em áreas comerciais, políticas, dentre outras (BARBOSA, 2012).

Entretanto, esta única rede social tem gerado diariamente uma maciça quantidade de dados, o que torna o trabalho dos engenheiros e cientistas de criar formas eficientes de armazenar, processar e recuperar informação, um grande desafio.

Ainda que um sistema de cluster demonstre ser uma boa alternativa para dar suporte ao processamento de grandes quantidades de informação, os tradicionais modelos de armazenamento de arquivos, baseados em um sistema Cliente-Servidor, demonstra ser eficiente apenas em clusters de baixa escala, já que a medida que o número de computadores acoplados ao sistema aumenta, é criado uma vazão de requisições para acesso a informações grande o suficiente para comprometer o desempenho do sistema (KUSZERA, 2010). Ou seja, aplicações paralelas intensivas em dados, sob um sistema de clusters de grande porte apresentam limitações de desempenho, ao mesmo tempo que geram baixa escalabilidade e throughput.

Uma alternativa que surgiu para tratar os problemas citados acima foi a utilização de sistemas de arquivos distribuídos. Neste modelo, os arquivos que armazenam os dados são divididos entre os nós do cluster, possibilitando o acesso em paralelo de diferentes partes de um mesmo arquivo. Esta estratégia permite aumentar a vazão de dados ao mesmo tempo que promove ampliação da escalabilidade do sistema (KUSZERA, 2010). Entretanto, a complexidade desta arquitetura gera um maior grau de dificuldade para instalação, configuração e gerenciamento do sistema como um todo, dificultando a produção neste ambiente de trabalho (KUSZERA, 2010).

Nesse sentido, este trabalho objetiva investigar sistemas de arquivos distribuídos que permitam da suporte ao a trabalhos que demandam uso de uma quantidade maciça de dados, como o de análise de sentimentos, visando compreender melhor seu funcionamento e arquitetura. 


\section{MATERIAL E MÉTODOS OU METODOLOGIA (ou equivalente)}

A pesquisa fará uso dos computadores e equipamentos instalado no Laboratório de Computação de Alto Desempenho (LaCAD), localizado na sala I12 do LABOTEC3. A metodologia utilizada envolverá:

1. Estudo das plataformas de sistemas de arquivos distribuídos mais reconhecidas no mercado;

2. Experimentos e comparação de desempenho para selecionar o sistema mais mais eficientes e adequado à instalação nos clusters do LaCAD;

3. Apresentação dos resultados através de palestras ou publicação de artigos.

\section{RESULTADOS E/OU DISCUSSÃO (ou Análise e discussão dos resultados)}

Foi realizada uma revisão da literatura em artigos científicos a fim de encontrar quais são os Sistemas de Arquivos Distribuídos (Do inglês -Distributed FIle System (DFS) ) gratuitos disponíveis mais utilizadas. Nesta pesquisa, foram encontrados exemplos como Hadoop (SHVACHOKO, 2010) e Lustre (KUSZERA, 2010).

o Lustre é um sistema de arquivos distribuídos com grande visibilidade e utilização entre pesquisadores e centros de pesquisa (KUSZERA, 2010) e por esta razão, ele foi a plataforma escolhida para a realização da pesquisa. Apesar de existirem outros sistemas de arquivos distribuídos o Lustre se sobressai em questão de visibilidade. O Lustre tem por objetivo possibilitar alta escalabilidade, eficiência e redundância, permitindo a criação de sistemas com grande escalabilidade de armazenamento e vazão de dados (KUSZERA, 2010).

O Rocks é o sistema padrão para a montagem, instalação e configuração de clusters do Laboratório de Computação de Alto Desempenho (LaCAD) da Universidade Estadual de Feira de Santana (UEFS). Desta maneira, o laboratório já possui domínio sobre a usabilidade, instalação e configuração deste sistema. Uma vez que já se tenha definido a plataforma padrão de cluster utilizada no laboratório, o objetivo desta pesquisa foi instalarar um DFS que permitisse gerir de forma eficiente uma quantidade maciça de dados em um cluster baseado no Rocks. Desta forma, foi decidido instalar o Lustre no Rocks na versão 6.5 .

Durante a pesquisa, descobriu-se que o primeiro passo para a instalação do lustre é a atualização da biblioteca e2fsprogs, que é um pacote de utilitários necessários para utilização de sistemas de arquivo no formato ext2, ex3 e ex4, que são sistemas de arquivos para o Kernel do Linux (CAO, 2005) e o Lustre é dependente do formato ext2. Entretanto, o Lustre depende de uma versão específica desta biblioteca que é superior a versão que vem instalada nativamente no Rocks na versão 6.5. Descobriu-se também que o Rocks não possui em seus repositórios uma atualização para a biblioteca e2fsprogs e por esta razão, sua atualização deveria ser realizada manualmente e assim o próximo passo da pesquisa foi descobrir como realizar esta atualização.

Uma das formas pensadas para realizar a atualização da biblioteca e2fsprogs foi pelo download de seus pacotes no formato RPM e instalação via terminal. Entretanto, este método foi bloqueado pelo Sistema Operacional (SO), pois o mesmo acusava que esta ação resultaria na presença de múltiplas versões da biblioteca e2fsprogs e isso acarretaria em problemas futuros. Outra alternativa existente foi uma edição nos arquivos dos repositórios de atualizações do Rocks para adicionar uma atualização para a biblioteca desejada o que teoricamente permitiria o sistema de atualização instalar automaticamente a versão da exigida pelo Lustre. Isso foi feito pela adição da seguinte instrução no repositório de atuação do Rocks: 
[e2fsprogs]

name $=$ CentOS-\$releasever - Ldiskfs

baseurl=https://downloads.hpdd.intel.com/public/e2fsprogs/1.42.13.wc6/el6/

gpgcheck $=0$

Esta ação também não funcionou, porque o SO acusou problemas de comunicação com o servidor onde os arquivos de atualizações da biblioteca e2fsprogs estavam localizados.

Uma vez que as opções de atualizações da biblioteca e2fsprogs foram esgotadas, foi usada a opção de instalar a versão mais atualizada possível do Rocks, porque ela traria versões mais atualizadas das bibliotecas e pacotes desejados. Esta versão não é a padrão utilizada no laboratório e deste modo, foi necessário dedicar tempo para pesquisar sua nova forma de instalação.

Durante a pesquisa, descobriu-se que Rocks na versão 7.0 mudou completamente a sua forma de instalação. Nas versões anteriores, os ROLLS, pacotes utilizados para instalar módulos do Rocks, localizavam-se junto ao CD de instalação. Com as mudanças na versão 7.0, os ROLLS são baixados durante o processo de instalação do SO. Como a infraestrutura de internet da UEFS é lenta, não foi possível baixar esses arquivos e com isso não foi possível instalar uma versão mais atualizada do sistema de gestão de cluster do LaCAD usando o procedimento padrão.

Uma alternativa pensada para solucionar este problema foi baixar os ROLLS fora da UEFS e arquivá-los em um dispositivo de memória. No momento da instalação, mudaria-se o local onde o instalador baixava os ROLLS para este dispositivo de memória e assim não seria necessário baixá-los novamente. Entretanto esta opção falhou, porque embora o sistema de instalação reconhecesse o dispositivo, ele não foi capaz de encontrar os arquivos armazenados no mesmo.

Não se desistiu de imediato da instalação do Lustre no Rocks porque foram encontrados na Internet instruções e tutoriais mostrando passos para a instalação do Lustre no Rocks. Entretanto, durante o decorrer do período de bolsa não se conseguiu um resultado positivo na reprodução dessas mesmas instruções e tutoriais. Uma das possibilidades que impediram a reprodução das instalações o Lustre na versão padrão Rocks do utilizada no laboratório, é a de que os hardwares que compõem os computadores dos clusters são antigos e isso influenciou na instalação dos arquivos necessários para o funcionamento do DFS selecionado para testes.

Uma vez que não foi encontrado uma forma alternativa que permitisse a instalação do Lustre no Rocks, foi iniciada uma outra pesquisa para a instalação do Lustre no CentOS em máquinas independentes, posteriormente interligando-as através de outro sistema que não fosse o Rocks. Instalar o Lustre no CentOS apresenta uma série de desvantagens se comparado com sua instalação no Rocks, como por exemplo, uma perda da automatização do processo de instalação, uma vez que é necessário instalar manualmente o $\mathrm{SO}$ em todos os computadores que farão parte do cluster do DFS. Outra desvantagem é que todos os clientes Lustre deverão ser instalados manualmente. Além disso, pode-se citar ainda como desvantagem é que a configuração da rede que permite a comunicação entre os computadores deve ser montada manualmente, enquanto que no Rocks isso é automatizado.

O CentOS na versão 7.0, assim como o Rocks 6.5, não possuía a biblioteca e2fsprogs na versão mínima exigida pelo Lustre, porém com uma edição do repositório de atualizações do $\mathrm{SO}$, adicionando as mesmas instruções descritas acima para o ROCKS, foi possível atualizar com sucesso a biblioteca para a versão desejada. Assim, 
uma vez que as dependências exigidas pelo Lustre foram completas, foi possível instalá-lo na no CentOS na versão 7.0

Nesse sentido, com a finalização desta pesquisa foi possível realizar um estudo sobre os possíveis Sistemas de Arquivos Distribuídos de domínio público disponíveis e selecionar o Lustre para uso futuro do Laboratório. Com isso, foi trazido para o LaCAD conhecimento acerca do funcionamento e instalação do Lustre no Centos na versão 7.0. Deste modo, esta pesquisa contribuiu para diversificar as linhas de pesquisa no laboratório uma vez que viabiliza a realização e ampliação de trabalhos em áreas que demandam Sistemas de arquivos Distribuídos.

\section{CONSIDERAÇÕES FINAIS (ou Conclusão)}

O objetivo desta pesquisa foi Avaliar plataformas de sistemas de arquivos distribuídos para suporte a pesquisa de análise de sentimentos e mineração de dados em um ambiente de alto desempenho, ao mesmo tempo que Obter maiores informações sobre instalação, gerenciamento, e manutenção de sistemas de arquivos distribuídos. Para isso, a pesquisa fez uso dos computadores e equipamentos instalado no Laboratório de Computação de Alto Desempenho (LaCAD), localizado na sala I12 do LABOTEC3.

O resultado do trabalho foi comprometido pela má infraestrutura da Internet que não permitiu o download dos $R O L L S$ necessários para a instalação de uma versão mais atualizada do sistema utilizado para a gestão dos Clusters no laboratório. Uma vez que não foi possível baixar esses mesmo ROLLS, a instalação do ROCKS na versão 7.0 não foi finalizada.

A instalação do Lustre foi realizada no CentOS na versão 7.0. Apesar de atividades planejadas como medição de desempenho e testes de funcionalidade não terem sido realizadas, uma vez que não houve tempo hábil para tal, consequência da dedicação de mais tempo à atividades de pesquisa e instalação do Lustre no Rocks, foi possível realizar um estudo sobre plataformas de Sistemas de Arquivos Distribuídos e trazer para o LaCAD conhecimento sobre o funcionamento e instalação do Lustre no CentOS na versão 7.0.

\section{REFERÊNCIAS}

SHVACHOKO, K. KUANG, H. RADIA, S . CHANSLER, R . "The Hadoop Distributed File System”, Yahoo!, Sunnyvale, California USA, 2010.

KUSZERA, E. M. AVALIANDO O SISTEMA DE ARQUIVOS LUSTRE COM USO DE CARGAS DE TRABALHO DE APLICAÇÕES PARALELAS, 2010, 90f. Tese (Mestrado em Ciência da Computação) - Pontifícia Universidade Católica do Rio Grande do Sul. Porto Alegre, 2010.

CAO, Mingming et al. State of the art: Where we are with the ext3 filesystem. In: Proceedings of the Ottawa Linux Symposium (OLS). 2005. p. 69-96.

BARBOSA, L.FENG, J Robust Sentiment Detection on Twitter from Biased and Noisy Data, Beijing, August 2012. 